\title{
An Improved Model for Headway-Based Bus Service Unreliability Prevention with Vehicle Load Capacity Constraint at Bus Stops
}

\author{
Weiya Chen, ${ }^{1,2}$ Chunhua Yang, ${ }^{1}$ Fenling Feng, ${ }^{2}$ and Zhiya Chen ${ }^{2}$ \\ ${ }^{1}$ School of Information Science and Engineering, Central South University, Changsha 410083, China \\ ${ }^{2}$ School of Traffic and Transportation Engineering, Central South University, Changsha 410075, China \\ Correspondence should be addressed to Fenling Feng, ffl0731@163.com
}

Received 29 September 2012; Revised 22 November 2012; Accepted 27 November 2012

Academic Editor: Wuhong Wang

Copyright $(02012$ Weiya Chen et al. This is an open access article distributed under the Creative Commons Attribution License, which permits unrestricted use, distribution, and reproduction in any medium, provided the original work is properly cited.

\begin{abstract}
This paper presents an improved model for improving headway-based bus route service reliability at bus stops using real-time preventive operation control, taking into account dynamic interaction among random passenger demand, stochastic driving conditions of route segments, and vehicle load capacity constraint. In this model, the real-time information of passenger demand and vehicle operation is involved to predict the imminent unacceptable headway deviation, in the case of which some in-time preventive control strategies are deployed according to the given control rules. As a case study, a single fixed bus route with high-frequency services was simulated and different scenarios of real-time preventive operation control were performed. Headway adherence and average passenger wait time were used to measure bus service reliability. The results show that the improved model is closer to the real bus route service, and using real-time information to predict potential service unreliability and trigger in-time preventive control can reduce bus bunching and avoid big gap.
\end{abstract}

\section{Introduction}

Giving priority to the development of urban public transit is becoming the common view on reducing urban traffic jam and improving urban travel efficiency [1]. But it is not just a policy issue to attract more and more people to choose transit for travelling. A challenging problem faced by the government, researchers, and transit agencies is how to provide better transit service by using up-to-date technologies.

Reliability is one of the most important attributes of quality of transit service and always the top concerned issue for both passengers and transit agencies [2, 3]. From the perception of the passengers, service unreliability means more average wait time, which is 
identified by Welding's assertion that the more regular service means the lower average wait time for potential passengers, especially on high-frequency bus routes with random passenger demand [4]. For transit agencies, service delays and disruptions have a real monetary cost in terms of lower utilization of vehicles and operators, which account for $3-5 \%$ of operating and vehicle costs by conservative estimate $[5,6]$. In terms of the causes of unreliability, running time variability and passenger demand fluctuation are generally noted to be significant factors for service unreliability [7, 8]. Moreover, the initial headway irregularity, either at the beginning or the mid-route, will propagate downstream and this kind of propagation tends to worsen passenger load fluctuation and contributes to worse unreliability downstream $[9,10]$.

In order to improve bus service reliability, the provision of real-time information technologies has been recognized as a persuasive strategy in more and more countries. In recent days, real-time information technologies like automatic vehicle location (AVL) and automatic passenger count (APC) are used to monitor service and passenger demand, based on which different operation control strategies are adopted to control bus operation [11-15]. From the existing research, most of the control strategies were studied to restore service reliability after the occurrence of disruption [16, 17]. Considering real-time information collected by the advanced public transit system, however, it is expected to forewarn big headway deviation from the beginning of the route service and adopt some immediate preventive action to avoid potential service irregularity [18-21]. In addition, in a rich information environment, model-based intelligent vehicle systems would help drivers to achieve the effectiveness of the vehicle operation control $[22,23]$. At this point, an effective model for decision-making using real-time information is the key issue for real-time bus operation control.

In [20], disregarding the vehicle load capacity constraint, we developed a simulation model for fixed-route transit service unreliability prevention based on AVL-APC data, whose effectiveness was extensively demonstrated on a circular bus route [21]. However, in the real world, vehicle load capacity is a very important factor affecting bus service reliability from the perspective of passengers. Based on the main framework of the model presented in [20], this paper develops an improved model for improving bus service reliability by using instantaneous prediction and preventive control strategies on high-frequency bus route, considering the dynamic interaction among random passenger demand, stochastic driving conditions of route segments and the vehicle load capacity constraint. In addition, it presents a set of decision-making rules for the potential intelligent dispatching module.

This rest of the paper is organized as follows. The next section presents the measures of headway-based transit service reliability. In Section 3, a vehicle-load-capacity-constrained preventive model is developed based on the main model framework in [20]. Section 4 presents a Monte Carlo simulation with the model embedded, which applies real-time preventive control approach to a simple fixed bus route with high-frequency services. Conclusions and future work are provided in Section 5.

\section{Measures of Headway-Based Bus Service Reliability}

\subsection{Coefficient of Variation of Headways $\left(C_{v h}\right)$}

On high-frequency bus routes, passengers generally arrive at stops randomly disregarding the schedule. Therefore, headway adherence is the most important index for measuring service reliability. In the Transit Capacity and Quality of Service Manual (TCQSM) [24], the 
Table 1: The level of service classified by $C_{v h}$ for fixed-route bus service.

\begin{tabular}{lccc}
\hline LOS & $C_{v h}$ & $P\left(h_{i}>0.5 h\right)$ & Comments \\
\hline A & $0.00-0.21$ & $\leq 1 \%$ & Service provided like clockwork \\
B & $0.22-0.30$ & $\leq 10 \%$ & Vehicle slightly off headway \\
C & $0.31-0.39$ & $\leq 20 \%$ & Vehicle often off headway \\
D & $0.40-0.52$ & $\leq 33 \%$ & Irregular headways, with some bunching \\
E & $0.53-0.74$ & $\leq 50 \%$ & Frequent bunching \\
F & $\geq 0.75$ & $>50 \%$ & Most vehicle bunched \\
\hline Note: (a) The probability $P$ means that a given transit vehicle's headway $h_{i}$ will be off headway by more than one-half the \\
scheduled headway $h$. \\
(b) Applies to routes with headways of 10 minutes or less. \\
Source: transit capacity and quality of service manual-2nd edition.
\end{tabular}

measure is based on the coefficient of variation of headways $\left(C_{v h}\right)$ of transit vehicles serving a fixed route arriving at a stop and is calculated as follows:

$$
C_{v h}=\frac{\text { Standard deviation of headway deviations }}{\text { mean schedule headway }} .
$$

For fixed-route high-frequency bus services, the level of service (LOS) is classified according to the coefficient of variation of headways (see Table 1).

\subsection{Average Passenger Wait Time}

Welding's average passenger wait time is also widely used to measure headway-based transit service reliability, which is a function of the mean and variance of the headway [3]:

$$
E(W)=\frac{1}{2}\left(E(H)+\frac{\operatorname{Var}(H)}{E(H)}\right) .
$$

In (2.2), $E(W)$ is the expected passenger wait time, $E(H)$ is the expected headway, and $\operatorname{Var}(H)$ is the variance of the headway. According to (2.2), when $E(H)$ is a constant, the smaller $\operatorname{Var}(H)$ means the less average passenger wait time $E(W)$.

\section{Model Development}

In order to improve bus service reliability, static preventive control strategies include route planning, scheduling, and fleet and labor management [25]. On dynamic bus operation control, an in-time preventive strategy could be adopted to avoid big headway deviation before the imminent bus bunching or gap, which requires real-time bus service information for in-time decision making of operation control. In terms of headway-based preventive control, it is expected to keep $\operatorname{Var}(H)$ in (2.2) close to zero as possible. Therefore, as done in [20], the prediction and forewarning of potential service irregularity using real-time information and the immediate action to prevent the headway from deviating too much is the underlying principle of this improved model. 
Before the improved preventive model is formulated, we use a single fixed bus route for description. General assumptions are made and the notations used in this paper are listed below.

(a) Vehicles arrive at each stop frequently (e.g., headways of 5 minutes), making it proper to assume random passenger arrivals at each stop. Moreover, the arrival rate and alighting fraction at a given stop do not change over the observed time period.

(b) When a vehicle arrives at a stop, boarding and alighting of passengers can occur simultaneously (usually for vehicles with a front door for boarding and a rear door for alighting). The average boarding time and alighting time of each passenger are constant for simplicity.

(c) The dwell time of a vehicle at a given stop is the maximum of the boarding time and the alighting time.

(d) The running time of a vehicle in between two stops is random, but still predictable and controllable to some extent.

(e) Considering the real-time information of passenger demand and vehicle operation, when a vehicle leaves the current bus stop, the dwell time and running time of its preceding vehicle, as well as its own leading headway and the on-board passenger number, are known for decision making of a preventive control strategy.

(f) Considering vehicle load capacity, those passengers who are not able to take the current fully loaded bus have to wait for the next bus. Vehicles are not allowed to overtake each other under preventive control.

A set of variables will be used for describing the interaction between the vehicles and passengers on this fixed bus route.

$H_{i k}=$ the leaving headway of the vehicle $i$ departing from the stop $k$;

$R_{i k}=$ the running time of the vehicle $i$ from stop $k-1$ to stop $k$;

$D_{i k}=$ the dwell time of the vehicle $i$ serving passengers' boarding and alighting at stop $k$;

$B_{i k}=$ the random number of passengers boarding the vehicle $i$ at stop $k$;

$U B_{i k}=$ the number of passengers who are not able to get on the vehicle $i$ at stop $k$ due its capacity constraint and full loading;

$A_{i k}=$ the random number of passengers alighting from the vehicle $i$ at stop $k$;

$L_{i k}=$ the number of on-board passengers of the vehicle $i$ when it departs from stop $k$;

$\lambda_{k}=$ the passenger arrival rate (passengers per minute) at stop $k$;

$\rho_{k}=$ the passenger alighting fraction of the on-board passengers at stop $k$;

$\alpha=$ the average alighting time for each passenger;

$\beta=$ the average boarding time for each passenger;

$C=$ the vehicle load capacity. 


\subsection{Dynamic Interaction between Passengers and Vehicles}

The route-level bus service system is a typical discrete dynamic system. The dynamic interaction between passengers and vehicles will be described based on the main model framework presented in [20]. The transit vehicles spend time on both traveling in between stops and dwelling at stops to serve passengers' boarding and alighting. The dwelling time of the vehicle $i$ at stop $k$ is decided by the maximum of the times of passengers' alighting and boarding. According to the assumption (b), $D_{i k}$ will be decided by

$$
D_{i k}=\max \left(\alpha \cdot A_{i k}, \beta \cdot B_{i k}\right) .
$$

Disregarding the vehicle load capacity $C, A_{i k}$ and $B_{i k}$ will be calculated or predicted by (3.2)

$$
\begin{gathered}
A_{i k}=\rho_{k} \cdot L_{i, k-1}, \\
B_{i k}=\lambda_{k} \cdot H_{i k} .
\end{gathered}
$$

Considering the constraint of the vehicle load capacity $C$, those passengers who are not able to get on the vehicle $i-1$ at stopk will have to wait for the vehicle $i$. Thus we will have $B_{i k}, U B_{i k}$, and $L_{i k}$ in (3.3) as

$$
\begin{gathered}
B_{i k}=\min \left(C-\left(1-\rho_{k}\right) \times L_{i, k-1}, \lambda_{k} \cdot H_{i k}+U B_{i-1, k}\right), \\
U B_{i k}=\max \left(0, \lambda_{k} \cdot H_{i k}+U B_{i-1, k}-C+\left(1-\rho_{k}\right) \times L_{i, k-1}\right), \\
L_{i k}=L_{i, k-1}-A_{i k}+B_{i k} .
\end{gathered}
$$

When the vehicle $i$ is departing from stop $k-1$, the number of on-board passengers $L_{i, k-1}$ could be observed according to the real-time information. Thus, it is appropriate to predict the minimum $D_{i k}$ based on the observed $L_{i, k-1}$, say, the vehicle $i$ will have to dwell at stop $k$ at least for a total alighting time $\left(\alpha \cdot \rho_{k} \cdot L_{i, k-1}\right)$, which can be formulized as (3.4).

$$
\left(D_{i k}\right)_{\min }=\alpha \cdot \rho_{k} \cdot L_{i, k-1}
$$

The boarding time $\left(\beta \cdot \lambda_{k} \cdot H_{i k}\right)$ will depend on the headway $H_{i k}$ with given $\beta$ and $\lambda_{k}$; while the headway $H_{i k}$ depends on the trajectories of transit vehicles. One step of iteration is as follows:

$$
\begin{gathered}
H_{i k}=H_{i, k-1}+\Delta R_{i k}+\Delta D_{i k}, \\
\Delta R_{i k}=R_{i k}-R_{i-1, k} \\
\Delta D_{i k}=D_{i k}-D_{i-1, k}
\end{gathered}
$$


where $\Delta R_{i k}$ is the difference in running times between bus $i$ and its preceding bus $i-1$ when they arrive at stop $k ; \Delta D_{i k}$ is the difference in dwell times between bus $i$ and its preceding bus $i-1$ when they dwell at stop $k$.

The dynamic iterative calculation of the headway $H_{i k}$ is as follows:

$$
H_{i k}=H_{i m}+\sum_{j=m+1}^{k} \Delta R_{i j}+\sum_{j=m+1}^{k} \Delta D_{i j}, \quad 0 \leq m \leq k-1, k>1 .
$$

Particularly, let $m=1$, the iteration will begin from the starting stop to the current stop $k$, then,

$$
H_{i k}=H_{i 1}+\sum_{j=2}^{k} \Delta R_{i j}+\sum_{j=2}^{k} \Delta D_{i j}, \quad k>1
$$

From (3.8) and (3.9), the headway $H_{i k}$ depends on the previous headways, the accumulated running time differences and dwell time differences, which present a theoretical proof for that the initial headway irregularity, either at the beginning or the mid-route, will propagate downstream. This kind of propagation is caused by the dynamic interactions between passengers and vehicles, and the worsen vehicle load fluctuation would contributes to the worse unreliability downstream, vice versa.

\subsection{Real-Time Preventive Bus Operation Control}

In a preventive model, the ideal headway $H_{i k}$ will be always expected to be kept close to the scheduled headway $H_{i}$ stop by stop $(k=1,2,3, \ldots, N)$ along the bus route. Mathematically, $H_{i k}$ is kept on the interval decided by $H_{i}$ plus or minus a permitted deviation $\varepsilon(\varepsilon>0)$ due to the stochastic operation environment as

$$
H_{i}-\varepsilon \leq H_{i k} \leq H_{i}+\varepsilon, \quad \varepsilon>0 .
$$

With the given vehicle load capacity and the initial headway, the headway $H_{i k}$ will mainly depends on the total running time difference and the total dwell time difference in between the first stop and the current stop $k$. When it comes to the operation control strategies, two options of control strategies will be decided. One option is bus holding strategy for controlling the vehicle dwell time; the other option is bus speed adjusting for controlling the vehicle running time. The bus speed adjusting strategy will be preferred on high-frequency bus route for two aspects of consideration. On one hand, the bus holding strategy works well for improving service reliability, but it has some disadvantages: wasting time of the on-board passengers, occupying the pick-up location at the bus station, delaying the arrival at the destination, and so forth [24]. On the other hand, the bus speed adjusting is acceptable and operational, which is benefited from the real-time traffic information technologies. Determining how to use the two strategies will be the key issue for the preventive control problem.

To illustrate this problem clearly, a simple example bus route is used (see Figure 1). On this single fixed bus route, the bus $i$ is leaving current stop $k-1$ to stop $k$; with the 


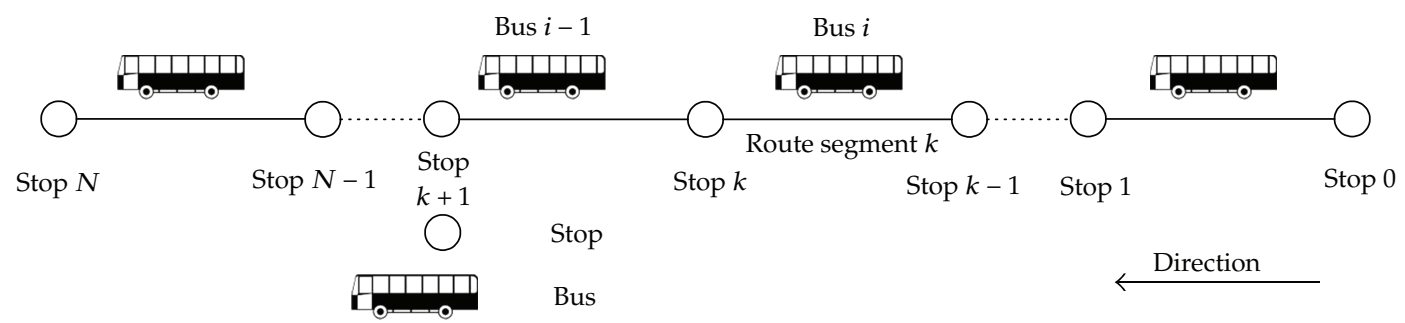

Figure 1: Example bus route.

observed $H_{i, k-1}, L_{i, k-1}, R_{i-1, k}$, and $D_{i-1, k}$ (according to the assumption(e)), we need to predict and suggest a reasonable running time $R_{i k}^{\prime}$ and $D_{i k}^{\prime}$ for bus $i$ to keep an acceptable $H_{i k}^{\prime}$. Determining an adaptive control strategy will follow the steps below.

Step 1 . To decide the predicted dwell time $\widetilde{D}_{i k}$. As discussed above, a minimum dwell time $\left(D_{i k}\right)_{\min }$ is the alighting time and can be calculated by (3.4); and only if the boarding time ( $\beta$. $\left.\lambda_{k} \cdot H_{i k}\right)$ is larger than the alighting time, the dwell time will equal to the boarding time. Here, we use a dummy variable $\varphi$ to determine the predicted dwell time $\tilde{D}_{i k}$, which is calculated as

$$
\widetilde{D}_{i k}=\varphi \cdot \alpha \cdot \rho_{k} \cdot L_{i, k-1}+(1-\varphi) \cdot \beta \cdot \lambda_{k} \cdot \bar{H}_{i}
$$

where

$$
\varphi= \begin{cases}1 & \text { for } \alpha \cdot \rho_{k} \cdot L_{i, k-1} \geq \beta \cdot \lambda_{k} \cdot \bar{H}_{i} \\ 0 & \text { otherwise }\end{cases}
$$

$\bar{H}_{i}$ is the expected headway of the vehicle $i$.

As discussed above, the vehicle speed control strategy will be firstly adopted and then will be the holding strategy. Therefore, the next step is to decide a reasonable running time.

Step 2. To decide a reasonable running time $R_{i k}^{\prime}$. A reasonable running time is decided jointly by the feasible running time and the acceptable running time. With the transfer of (3.5), an acceptable running time $\vec{R}_{i k}$ is given as follows:

$$
\begin{gathered}
\left(\bar{H}_{i}-\varepsilon-H_{i, k-1}+R_{i-1, k}+D_{i-1, k}\right)-\tilde{D}_{i k} \leq \vec{R}_{i k} \\
\leq\left(\bar{H}_{i}+\varepsilon-H_{i, k-1}+R_{i-1, k}+D_{i-1, k}\right)+\tilde{D}_{i k}
\end{gathered}
$$

A feasible running time $\stackrel{\leftarrow}{R}_{i k}$ is decided by the real-time traffic conditions in between stop $k-1$ and stopk. Along with the development of intelligent transportation technologies, it will be easy to get the real-time road traffic information, such as current traffic volume and speed. Here we still use a prediction way to decide the feasible running time $\overleftarrow{R}_{i k}$. It 
is acceptable to predict it based on $R_{i-1, k}$ or the statistical mean running time $\overline{R_{k}}$ and the standard deviation $\sigma$, respectively,

$$
\begin{aligned}
R_{i-1, k} \cdot\left(1-\delta_{1}\right) & \leq \overleftarrow{R}_{i k} \leq R_{i-1, k} \cdot\left(1+\delta_{2}\right) \\
\overline{R_{k}}-x \cdot \sigma & \leq \stackrel{\leftarrow}{R}_{i k} \leq \overline{R_{k}}+y \cdot \sigma .
\end{aligned}
$$

In (3.14), $\delta_{1}$ and $\delta_{2}$ are nonnegative percentile fractions. In (3.15), $x$ and $y$ are nonnegative parameters used to adjust the deviation and reflect the real-time traffic conditions. Considering the combination of (3.13) and (3.14) or (and) (3.15), a reasonable $R_{i k}^{\prime}$ will be located on the interval decided jointly by $\vec{R}_{i k}$ and $\overleftarrow{R}_{i k}$ :

$$
R_{i k}^{\prime} \in\left[\left(\vec{R}_{i k}\right)_{\min ^{\prime}}\left(\vec{R}_{i k}\right)_{\max }\right] \cap\left[\left(\overleftarrow{R}_{i k}\right)_{\min }\left(\overleftarrow{R}_{i k}\right)_{\max }\right]
$$

Those values over the interval in (3.16) are the suggested running time for the vehicle $i$ leaving for stop $k$. Theoretically, the interval is possible to be void, which means the acceptable $\vec{R}_{i k}$ can not be satisfied, either because the minimum $\vec{R}_{i k}$ is bigger than the maximum feasible running time $\stackrel{\leftarrow}{R}_{i k}$ or because the maximum $\vec{R}_{i k}$ is smaller than the minimum $\stackrel{\leftarrow}{R}_{i k}$. In practice, the former reason is impossible due to an infinite $\overleftarrow{R}_{i k}$; then the bus will be suggested to run at any $\vec{R}_{i k}$. The latter situation usually means the bad traffic conditions, and bus $i$ will be requested to run at the maximum feasible speed to prevent the gap. By using the theoretical analysis, we make some decision-making rules for the reasonable running time as follows:

(1) If $\left(\vec{R}_{i k}\right)_{\max } \leq\left(\overleftarrow{R}_{i k}\right)_{\min }$, then $R_{i k}^{\prime}=\left(\overleftarrow{R}_{i k}\right)_{\min } ;$

(2) If $\left(\vec{R}_{i k}\right)_{\min } \leq\left(\overleftarrow{R}_{i k}\right)_{\min }$ and $\left(\vec{R}_{i k}\right)_{\max } \geq\left(\overleftarrow{R}_{i k}\right)_{\min }$ and $\left(\vec{R}_{i k}\right)_{\max } \leq\left(\overleftarrow{R}_{i k}\right)_{\max }$, then $R_{i k}^{\prime} \in$ $\left[\left(\overleftarrow{R}_{i k}\right)_{\min },\left(\vec{R}_{i k}\right)_{\max }\right]$

(3) If $\left(\vec{R}_{i k}\right)_{\min } \leq\left(\overleftarrow{R}_{i k}\right)_{\min }$ and $\left(\vec{R}_{i k}\right)_{\max } \geq\left(\overleftarrow{R}_{i k}\right)_{\max }$, then $R_{i k}^{\prime} \in\left[\left(\overleftarrow{R}_{i k}\right)_{\min }\left(\overleftarrow{R}_{i k}\right)_{\max }\right]$;

(4) If $\left(\vec{R}_{i k}\right)_{\min } \geq\left(\overleftarrow{R}_{i k}\right)_{\min }$ and $\left(\vec{R}_{i k}\right)_{\max } \leq\left(\overleftarrow{R}_{i k}\right)_{\max }$, then $R_{i k}^{\prime} \in\left[\left(\vec{R}_{i k}\right)_{\min }\left(\vec{R}_{i k}\right)_{\max }\right]$;

(5) If $\left(\vec{R}_{i k}\right)_{\min } \geq\left(\overleftarrow{R}_{i k}\right)_{\min }$ and $\left(\vec{R}_{i k}\right)_{\min } \leq\left(\overleftarrow{R}_{i k}\right)_{\max }$ and $\left(\vec{R}_{i k}\right)_{\max } \geq\left(\overleftarrow{R}_{i k}\right)_{\max }$, then $R_{i k}^{\prime} \in$ $\left[\left(\vec{R}_{i k}\right)_{\min }\left(\overleftarrow{R}_{i k}\right)_{\max }\right]$;

(6) If $\left(\vec{R}_{i k}\right)_{\min } \leq\left(\overleftarrow{R}_{i k}\right)_{\max }$, then $R_{i k}^{\prime}=\left(\vec{R}_{i k}\right)_{\min }$.

\section{Simulation and Results}

\subsection{Parameters Description}

In order to clarify this model, the example bus route in Figure 1 is also used for the case study. This bus route has 11 stops which are scattered about in a fairly uniformity of running time, with buses being dispatched from stop 0 to stop 10 at 5-minute headway $(E(H)=5)$. The vehicle load capacity is 80 persons $(C=80)$. 
Table 2: Example of bus route parameters.

\begin{tabular}{lcccc}
\hline Stop & $\begin{array}{c}\lambda_{k} / \\
\text { Passengers/min }\end{array}$ & $\begin{array}{c}\rho_{k} / \\
\%\end{array}$ & $\begin{array}{c}E\left(R_{k}\right) / \\
\min \end{array}$ & $\begin{array}{c}E(H) / \\
\min \end{array}$ \\
\hline 0 & 1.0 & 0.0 & - & 5.0 \\
1 & 0.75 & 0.0 & 3 & 5.0 \\
2 & 0.75 & 0.1 & 3 & 5.0 \\
3 & 2.0 & 0.2 & 3 & 5.0 \\
4 & 2.0 & 0.25 & 3 & 5.0 \\
5 & 3.0 & 0.6 & 3 & 5.0 \\
6 & 1.5 & 0.25 & 3 & 5.0 \\
7 & 0.75 & 0.25 & 3 & 5.0 \\
8 & 0.5 & 0.5 & 3 & 5.0 \\
9 & 0.1 & 0.1 & 3 & 5.0 \\
10 & 0.0 & 1.0 & 3 & 5.0 \\
\hline
\end{tabular}

Each bus is expected to run in between two successive stops in 3 minutes $\left(E\left(R_{k}\right)=3\right)$, leading to a total expected running time of 30 minutes from stop 0 to stop 10 in one direction. The dwell time at each stop depends on the passenger demand. On high-frequency bus route, usually passenger arrival at each stop is a Poisson process and the number of passengers alighting at each stop is subject to a binomial probability distribution based on the current passenger load on the vehicle $[6,13]$. Here the passenger demand is characterized with a Poisson passenger arrival rate $\lambda_{k}$ and a binomial probability of passenger alighting fraction $\rho_{k}$. In addition, the boarding time and the alighting time per passenger are assumed to be 3.6 seconds and 1.8 seconds $(\alpha=0.06, \beta=0.03)$. Table 2 gives the parameters of the operating conditions of the example bus route.

\subsection{Simulation Scenarios}

For illustrative purposes, in a single simulation period 30 buses from stop 0 to stop 10 are observed and a total of 20 simulation runs are performed for four cases.

Case 1. Running time $R_{i k}$ is fluctuated with $\delta_{1}=\delta_{2}=0.4$ and $\varepsilon=60$ seconds.

Case 2. Running time $R_{i k}$ is fluctuated with $\delta_{1}=\delta_{2}=0.4$ and $\varepsilon=90$ seconds.

Case 3. Running time $R_{i k}$ is fluctuated with $\delta_{1}=\delta_{2}=0.2$ and $\varepsilon=60$ seconds.

Case 4. Running time $R_{i k}$ is fluctuated with $\delta_{1}=\delta_{2}=0.2$ and $\varepsilon=90$ seconds.

The initial conditions for each single simulation run includes: each bus leaves stop 0 precisely at its given headway, and the first bus is supposed to run in between two consecutive stops precisely with 3 minutes. The preventive strategies will be triggered by checking the real-time conditions following Steps 1 and 2 in Section 3.2. 
Table 3: The observed average headway, standard deviation of headways, and coefficient of variation of headways.

\begin{tabular}{|c|c|c|c|c|c|c|c|c|c|c|c|}
\hline Sce & narios & Stop 1 & Stop 2 & Stop 3 & Stop 4 & Stop 5 & Stop 6 & Stop 7 & Stop 8 & Stop 9 & Stop 10 \\
\hline \multirow{3}{*}{ Case 1} & Avg hdw & 5.0645 & 5.0964 & 5.1042 & 5.1091 & 5.1340 & 5.1497 & 5.1616 & 5.1694 & 5.1698 & 5.1568 \\
\hline & Std hdw & 0.5636 & 0.5900 & 0.5965 & 0.6000 & 0.6414 & 0.5741 & 0.5603 & 0.5238 & 0.5305 & 0.5290 \\
\hline & $C_{v h}$ & 0.1127 & 0.1180 & 0.1193 & 0.1200 & 0.1283 & 0.1148 & 0.1121 & 0.1048 & 0.1061 & 0.1058 \\
\hline \multirow{3}{*}{ Case 2} & Avg hdw & 5.1093 & 5.1919 & 5.2702 & 5.3087 & 5.3304 & 5.3670 & 5.4001 & 5.4170 & 5.4103 & 5.4173 \\
\hline & Std hdw & 0.8262 & 0.8295 & 0.8644 & 0.8379 & 0.9244 & 0.8189 & 0.7609 & 0.7592 & 0.7541 & 0.7280 \\
\hline & $C_{v h}$ & 0.1652 & 0.1659 & 0.1729 & 0.1676 & 0.1849 & 0.1638 & 0.1522 & 0.1518 & 0.1508 & 0.1456 \\
\hline \multirow{3}{*}{ Case 3} & Avg hdw & 5.1290 & 5.1933 & 5.2185 & 5.2655 & 5.2988 & 5.3456 & 5.3819 & 5.4005 & 5.3938 & 5.3968 \\
\hline & Std hdw & 0.5529 & 0.5221 & 0.5186 & 0.5188 & 0.5745 & 0.4960 & 0.4676 & 0.4147 & 0.4073 & 0.4078 \\
\hline & $C_{v h}$ & 0.1106 & 0.1044 & 0.1037 & 0.1038 & 0.1149 & 0.0992 & 0.0935 & 0.0829 & 0.0815 & 0.0816 \\
\hline \multirow{3}{*}{ Case 4} & Avg hdw & 5.1831 & 5.3577 & 5.4580 & 5.5623 & 5.6341 & 5.7012 & 5.7574 & 5.7952 & 5.8196 & 5.8311 \\
\hline & Std hdw & 0.6506 & 0.6836 & 0.7083 & 0.7497 & 0.7800 & 0.7011 & 0.6483 & 0.5651 & 0.5078 & 0.5089 \\
\hline & $C_{v h}$ & 0.1301 & 0.1367 & 0.1417 & 0.1499 & 0.1560 & 0.1402 & 0.1297 & 0.1130 & 0.1016 & 0.1018 \\
\hline
\end{tabular}

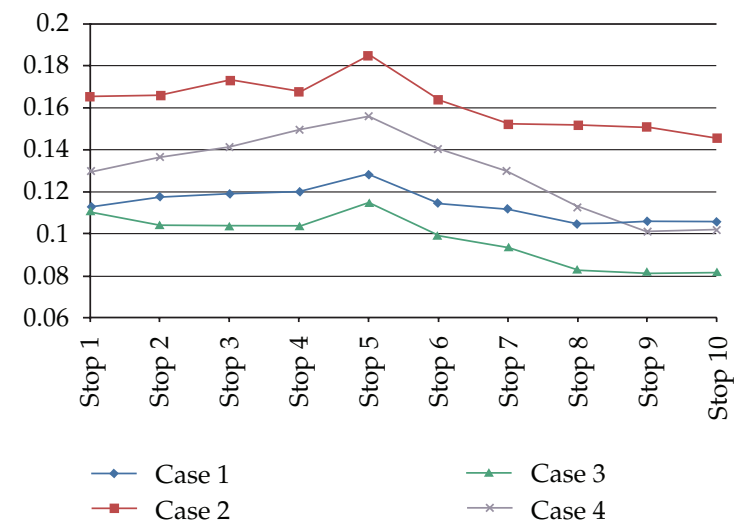

Figure 2: Stop-level changing of coefficient of variance of headways.

\subsection{Results and Discussion}

The two metrics described in Section 2 are used to measure service reliability. The first one is the observed average headway and the coefficient of variation of headways $\left(C_{v h}\right)$ at each stop. Table 3 gives the observed average headway (Avg hdw), the standard deviation of headways (std hdw), and the coefficient of variation of headways $\left(C_{v h}\right)$ for each case.

According to Table 3, the average headways in four cases are below 6 minutes and the coefficients of variation of headways are below 0.2. It also shows that, in all four cases, the biggest coefficients of variation of headways appear at stop 5 due to the highest probability of passenger boarding and alighting. The route-level changing of the coefficient of variation of headways is further shown in Figure 2. Compared with the results in [26], these results indicate good service unreliability prevention.

Comparing Cases 1 with 2, the running time fluctuation is the same with $40 \%$ of the scheduled running time, but the permitted headway deviation is different. From the simulation as shown in Figure 2, the coefficients of variation of headways at each stop in Case 1 are smaller than those of Case 2, which indicates that better service unreliability prevention 


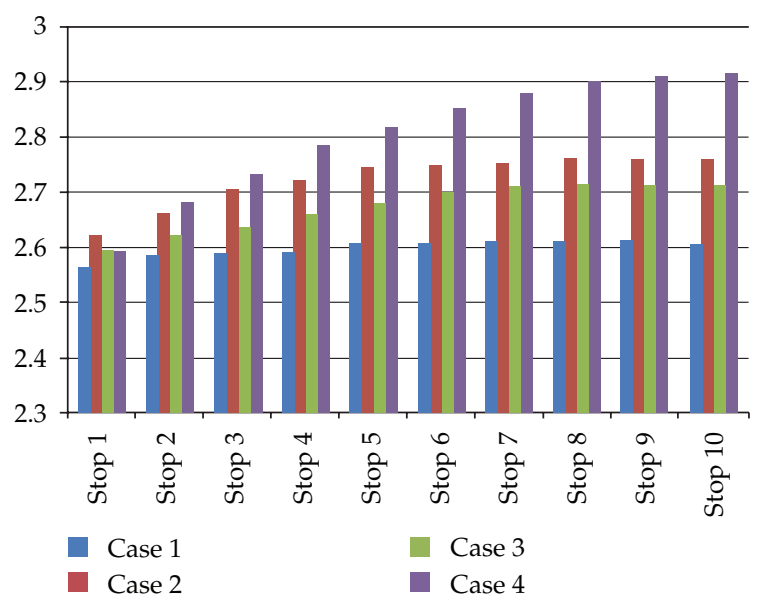

Figure 3: Average passenger wait time at each stop.

has been achieved in Case 1. The same situation appears when comparing Cases 3 with 4. These results suggest that the tougher request for keeping headway adherence promises better bus operation control. The practical reason might be that a bus driver may pay more attention to improving bus service reliability under strict performance evaluation.

Comparing Cases 1 with 3, the permitted headway deviation is the same with one minute, but the running time fluctuation is different. In Case 3, the lower limit of the running time fluctuation is half of that in Case 1 . The coefficients of variation of headways at each stop in Case 3 are smaller than those in Case 1. The same situation also appears when comparing Cases 2 with 4 . The results suggest that, requested by keeping the same permitted headway deviation, the smaller lower limit of the running time fluctuation is more likely to result in better headway regularity.

The other metric is average passenger wait time. Figure 3 shows the average passenger wait time at each stop in four cases.

As shown in Figure 3, the average passenger wait time at each stop is controlled at a level of less than 3 minutes in all four cases, but it still has a growing tendency. The best preventive effectiveness appears in Case 1, the worst in Case 4. Compared with the route-level changing of the coefficient of variation of headways, the growing tendency of the average passenger wait time is an interesting finding. This is noteworthy in that the intuition from the previous studies suggests that controlling one vehicle only by referring to its preceding vehicle might has a time lag of the travel time.

\section{Conclusions and Future Works}

This paper presents an improved preventive model for improving headway-based bus service reliability at bus stops, considering the constraint of the vehicle load capacity, as well as the dynamic nature of the random passenger demand and the stochastic road traffic conditions. Standing on this point, this model represents a more realistic approach to characterize the route-level bus service system and to utilize real-time information in a real-time decision-making context. As demonstrated in the simulation example, using some 
probability distributions of the running time and the constant passenger boarding time and alighting time, the model and the preventive control strategy are effective.

By using the simulation-based methodology, the model presented in this paper mainly presents a way of how to follow the steps and the decision-making rules to utilize the realtime information to make better decision. Nonetheless, a more practical decision-making supporting system or a more intelligent decision-making module will be more powerful and useful in the real application. In this sense, this model can be directly embedded in the advanced public transit system, assisting the dispatching center and the operators to utilize the real-time information including vehicle running time, speed, dwell time, passenger demand, and other unreliability causes to instantaneously predict and forewarn big headway deviation of high-frequency bus routes, based on which better decisions can be made regarding the use of preventive control strategies. This job has become our ongoing work, and a real bus route will be chosen in the city of Zhuzhou in China for testing the real effectiveness. With the real application and test, the improvements of this proposed methodology could be promised. More broadly, in terms of the preventive control strategies, the route-level multivehicle collaborative control based on synchronous simulation optimization is another important future work.

\section{Acknowledgments}

This work was supported by National Natural Science Foundation of China (NSFC) under Grants nos. 61203162 and 71171200. This work was also supported in part by the Postdoctoral Science Foundation of Central South University, the Freedom Explore Program of Central South University (Grant no. 721500036), and the Fundamental Research Funds for the Central Universities (Grant no. 2010QZZD021).

\section{References}

[1] M. Xu, A. Ceder, Z. Gao, and W. Guan, "Mass transit systems of Beijing: governance evolution and analysis," Transportation, vol. 37, no. 5, pp. 709-729, 2010.

[2] Y. Yin, W. H. K. Lam, and M. A. Miller, "A simulation-based reliability assessment approach for congested transit network," Journal of Advanced Transportation, vol. 38, no. 1, pp. 27-44, 2003.

[3] C. F. Daganzo and J. Pilachowski, "Reducing bunching with bus-to-bus cooperation," Transportation Research B, vol. 45, no. 1, pp. 267-277, 2011.

[4] P. I. Welding, "The instability of a close-interval service," Operation Research Quarterly, vol. 8, no. 3, pp. 133-148, 1957.

[5] M. H. Abkowitz, R. Slavin, L. Waksman, and N. W. Englisher, Transit Service Reliability, United States Department of Transportation, Washington, DC, USA, 1978.

[6] M. D. Hickman, "An analytic stochastic model for the transit vehicle holding problem," Transportation Science, vol. 35, no. 3, pp. 215-237, 2001.

[7] M. H. Abkowitz and I. Enelstin, "Factors affecting run time on transit routes," Transportation Research A, vol. 17, no. 2, pp. 107-113, 1983.

[8] L. C. Cham, Understanding Bus Service Reliability: A Practical Framework Using AVL/APC Data, Massachusetts Institute of Technology, Cambridge, Mass, USA, 2006.

[9] J. G. Strathman, T. J. Kimple, and S. Callas, Headway Deviation EffectS on BuS PaSSenger LoadS: AnalySiS of Tri-Met'S Archived AVL-APC Data, Portland State University, Portland, Ore, USA, 2003.

[10] Z. R. Peng, E. D. Lynde, and W. Y. Chen, Improving Service Restoration Using Automatic Vehicle Location, University of Wisconsin-Milwaukee, Milwaukee, Wis, USA, 2008.

[11] P. G. Furth, B. J. Hemily, H. J. Muller, and J. G. Strathman, Uses of Archived AVL-APC Data to Improve Transit Performance and Management: Review and Potential, Transportation Research Board, Washington, DC, USA, 2003. 
[12] A. Shalaby and A. Farhan, "Prediction model of bus arrival and departure times using AVL and APC data," Journal of Public Transportation, vol. 7, no. 1, pp. 41-61, 2004.

[13] I. E. Moses, A Transit Route Simulator for the Evaluation of Control Strategies Using Automatically Collected Data, Massachusetts Institute of Technology, Cambridge, Mass, USA, 2005.

[14] C. A. Pangilinan, N. Wilson, and A. Moore, Bus Supervision Deployment Strategies and the Use of RealTime AVL for Improved Bus Service Reliability, Transportation Research Board, Washington, DC, USA, 2008.

[15] A. M. El-Geneidy, J. Horning, and K. J. Krizer, Analyzing Transit Service Reliability Using Detailed Data from Automatic Vehicular Locator Systems, Transportation Research Board, Washington, DC, USA, 2008.

[16] J. G. Strathman, T. J. Kimpel, and K. J. Dueker, "Bus transit operations control: review and an experiment involving Tri-Met's automated bus dispatch system," Journal of Public Transportation, vol. 41, no. 1, pp. 1-26, 2001.

[17] A. Sun and M. Hickman, "The holding problem at multiple holding stations," Lecture Notes in Economics and Mathematical Systems, vol. 600, pp. 339-359, 2008.

[18] C. F. Daganzo, "A headway-based approach to eliminate bus bunching: systematic analysis and comparisons," Transportation Research B, vol. 43, no. 10, pp. 913-921, 2009.

[19] Y. Hadas and A. Ceder, "Optimal coordination of public-transit vehicles using operational tactics examined by simulation," Transportation Research C, vol. 18, no. 6, pp. 879-895, 2010.

[20] W. Y. Chen and Z. Y. Chen, "A simulation model for transit service unreliability prevention based on AVL-APC data," in Proceedings of the International Conference on Measuring Technology and Mechatronics Automation (ICMTMA '09), vol. 2, pp. 184-188, April 2009.

[21] W. Y. Chen and Z. Y. Chen, "A bus-following model for preventing service unreliability on a circular bus route," in Proceedings of the 2nd International Conference on Intelligent Computing Technology and Automation (ICICTA '09), vol. 3, pp. 425-428, October 2009.

[22] W. .H Wang, H. W. Guo, H. Bubb, and K. Ikeuchi, "Numerical simulation and analysis procedure for model-based digital driving dependability in intelligent transport system," KSCE Journal of Civil Engineering, vol. 15, no. 5, pp. 891-898, 2011.

[23] W. H. Wang, Vehicle'S Man-Machine Interaction Safety and Driver ASSiStance, China Communications Press, Beijing, China, 2012.

[24] KITTELSON \& ASSOCIATES, Inc. and KFH Group, Inc., Transit Capacity and Quality of Service Manual, Transportation Research Board, Washington, DC, USA, 2nd edition, 2003.

[25] M. A. Turnquist, "Strategies for improving reliability of bus transit service," Transportation Research Record, vol. 818, pp. 7-13, 1981.

[26] W. Y. Chen and Z. Y. Chen, "Service reliability analysis of high frequency transit using stochastic simulation," Journal of Transportation Systems Engineering and Information Technology, vol. 9, no. 5, pp. 130-134, 2009. 


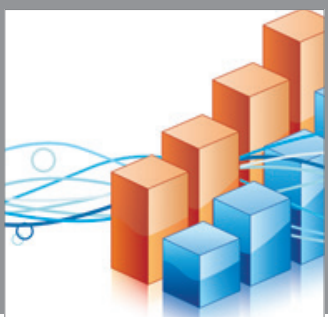

Advances in

Operations Research

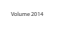

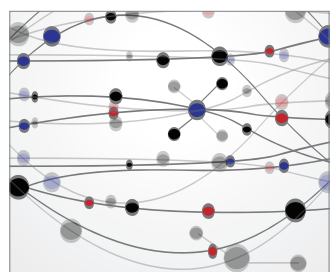

\section{The Scientific} World Journal
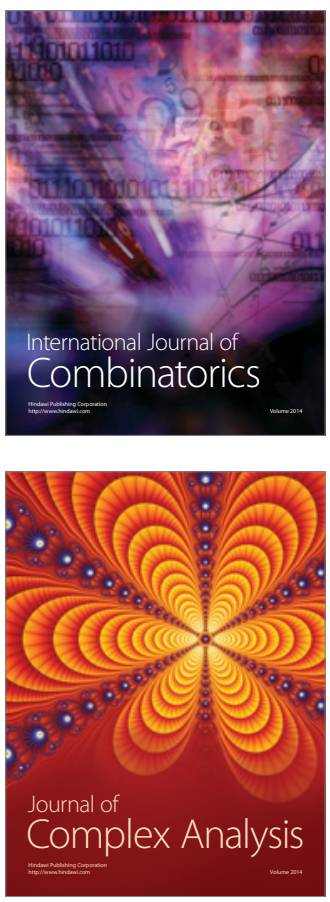

International Journal of

Mathematics and

Mathematical

Sciences
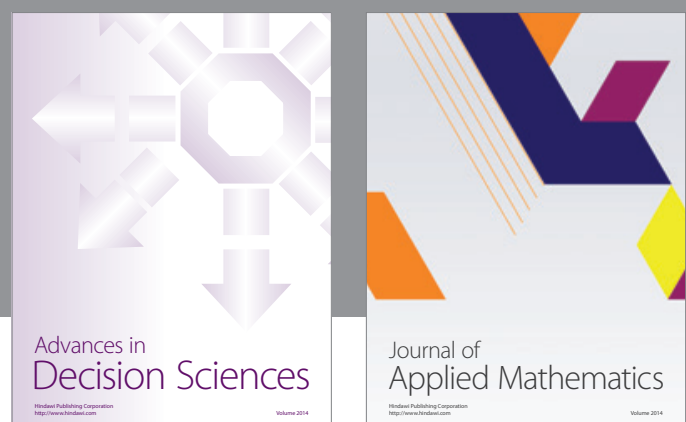

Journal of

Applied Mathematics
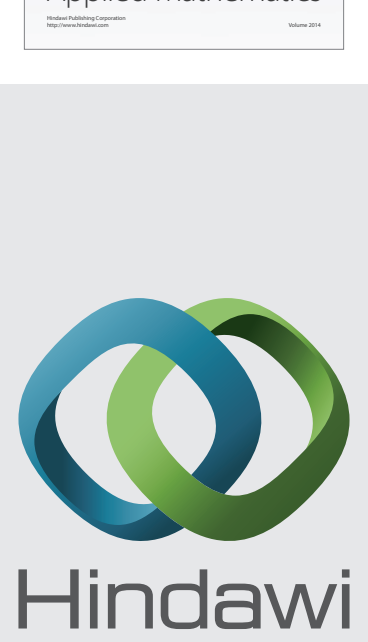

Submit your manuscripts at http://www.hindawi.com
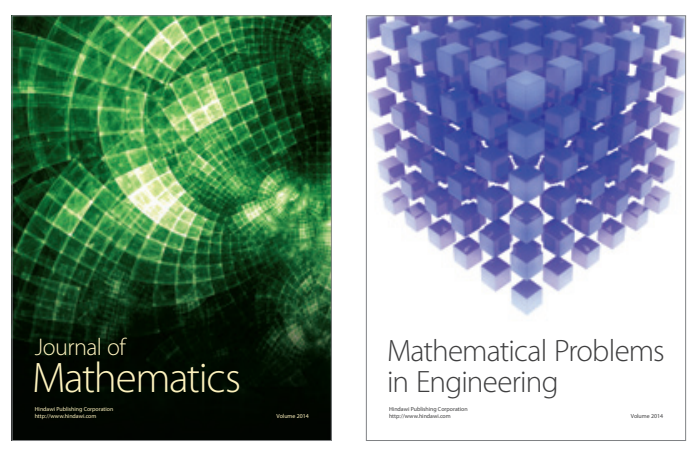

Mathematical Problems in Engineering
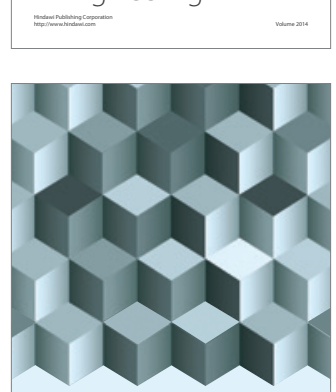

Journal of

Function Spaces
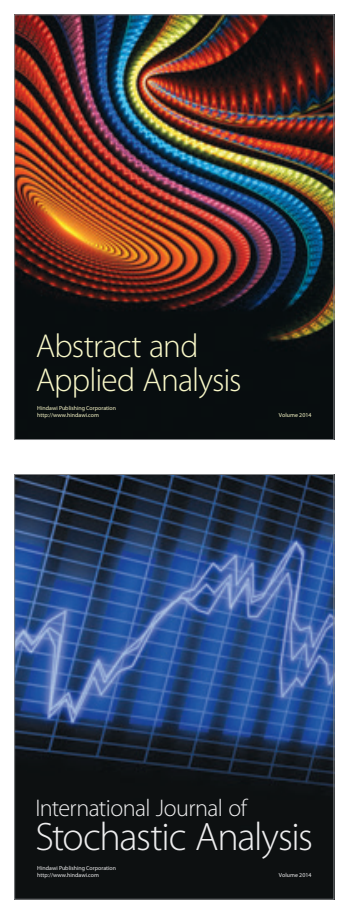

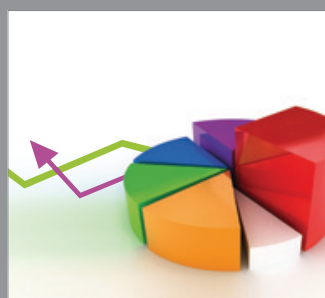

ournal of

Probability and Statistics

Promensencen
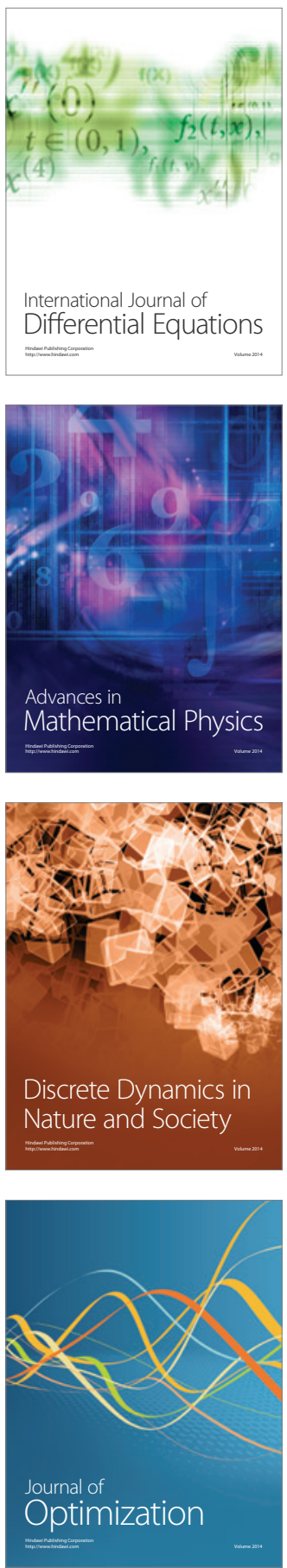